\title{
La dinámica del subdesarrollo y su relación con el deterioro ambiental
}

\section{The dynamics of underdevelopment and its relationship to environmental deterioration}

\author{
Carlos Dionisio Pérez-Blanco*
}

\begin{abstract}
Are developed countries responsible for environmental deterioration? Do developing countries have the right to pollute more? The paper refuses this hypothesis and shows that a dynamic of growth aimed towards consistent development in low income areas has to be necessarily sustainable; otherwise, it can undermine a country's development path. The paper surveys mainstream development economics theory and argues that there is a consistent relation between development and environmental standards. It is pointed out the problem that many underdeveloped countries face when trying to get out of a low income development trap and the consequences of these traps over its wealth (which includes natural capital); this wealth is precisely the main factor determining the capacity of a country to generate income in the future. Thus, an in depth knowledge of development traps dynamics is needed to prevent environmental deterioration from happening.
\end{abstract}

Keywords: big push, Kuznets curve, dual economy, institutional quality, genuine wealth.

\section{Resumen}

¿Son los países desarrollados los culpables del deterioro ambiental?, y por tanto, ¿tienen los países subdesarrollados derecho a contaminar más? En este artículo se rebate esta tesis para mostrar que una dinámica de crecimiento que conduce al desarrollo de un territorio de renta baja debe ser por necesidad sostenible, si este país no desea liquidar sus posibilidades de desarrollo. Partiendo de las principales aportaciones teóricas de la economía del desarrollo, el artículo defiende la reciprocidad entre desarrollo y calidad ambiental. Se señala la dificultad que tienen los países subdesarrollados para abandonar un equilibrio de bajo nivel, y lo que esto implica en su riqueza (en concreto su capital ambiental), que es la que en última instancia determina su capacidad de generar renta en el futuro. Se requiere por tanto una comprensión profunda de la dinámica del subdesarrollo para frenar el deterioro ambiental.

Palabras clave: big push, curva de Kuznets, economía dual, calidad institucional, riqueza genuina.

* Consejo Superior de Investigaciones Científicas en Madrid, España. Correo-e: rage_dioni@ hotmail.com. 


\section{Introducción}

Se ha planteado en diversos foros internacionales (económicos o de otra índole) la responsabilidad de los países desarrollados ${ }^{1}$ para con los que están en vías de desarrollo, en lo económico y en lo ambiental; éste es un intento de justificar o legitimar el derecho de las economías en vías de desarrollo a contaminar comparativamente más, de manera que tengan las mismas oportunidades que tuvieron en su día los países desarrollados. El argumento se basa en un principio de justicia restaurativa que implícitamente reconoce que un mayor derecho a contaminar es capaz de fomentar per se el crecimiento económico.

En este artículo, a través de una revisión bibliográfica de la economía ambiental, se defiende la postura de que, si bien una regulación laxa en la gestión de los recursos naturales puede generar incrementos de la renta en el corto y mediano plazos, una política basada exclusivamente en la explotación de recursos naturales no genera crecimiento y además tiene efectos negativos sobre la riqueza natural (capital). El capital es la fuente de crecimiento y el indicador adecuado para medir el bienestar (Dasgupta, 2009), por lo que una depreciación del capital natural afecta de manera negativa las posibilidades de desarrollo y el bienestar de la economía.

A partir de esta base se cuestiona el modelo de crecimiento extensivo que se está llevando a cabo en determinados países y regiones en desarrollo, que en determinados casos está liquidando la riqueza de estas regiones y sus futuras posibilidades de desarrollo (sobreexplotación de los recursos hídricos y mix energético no renovable en los nuevos países industrializados; desertización y sobreexplotación de recursos forestales en los Estados fallidos africanos; deforestación en los grandes Estados emergentes). De lo anterior se desprende que el debate inicial está mal formulado: no debemos cuestionarnos si los paises pobres deben tener el mismo derecho a contaminar (o más) que los ricos (suponiendo que este derecho a contaminar existiera), sino si un modelo de crecimiento agresivo con el medio reportará a aquéllos algún beneficio más allá del corto plazo o por el contrario condicionará sus posibilidades de desarrollo futuro.

Aquí se defiende la existencia de una relación circular entre desarrollo y sostenibilidad basada en la existencia de una curva de Kuznets ambiental, ${ }^{2}$

${ }^{1}$ Si bien no existe una división estricta ni una clasificación oficial que distinga entre países desarrollados y en vías de desarrollo, seguimos el esquema adoptado por la onU y consideramos países desarrollados a Japón, Canadá, Estados Unidos, Australia, Nueva Zelanda y a los de Europa (en las estadísticas de comercio internacional también se incorpora a Israel y Sudáfrica) (onU, 2008); el resto de países (nuevos países industrializados, economías en transición, grandes Estados emergentes y Estados fallidos) los consideramos economías en vías de desarrollo.

${ }^{2}$ Este término, propuesto por primera vez por Grossman y Krueger (1991), presenta una relación en forma de $U$ invertida entre crecimiento y deterioro ambiental. En las fases iniciales del desarrollo 
ampliamente debatida y documentada (Grossman y Krueger, 1991; Dasgupta et al., 2002; Harbaugh et al., 2002; Syed y Mohammad, 2004; ONU, 2005). Una región debe ser lo suficientemente respetuosa con el medio ambiente en sus primeras etapas de desarrollo (las más contaminantes) como para garantizar una cantidad de recursos naturales que faciliten alcanzar un desarrollo de alto nivel en las etapas intermedias y finales. En este proceso es necesaria una serie de factores institucionales, sociales, de coyuntura internacional, etc., que abordamos a lo largo de este trabajo.

El artículo tiene el siguiente esquema: el primer apartado aborda los distintos estadios del desarrollo, de la sociedad tradicional a la economía dual y el big push que conduce a un desarrollo de alto nivel; posteriormente se examina la maldición de los recursos naturales y se señala el factor institucional como aspecto detonante; en la tercera parte se presentan otros factores que afectan el crecimiento y sus efectos en la calidad ambiental; en el cuarto apartado se sintetizan algunas de las medidas paliativas del deterioro ambiental, con vigencia ante trampas de pobreza; finalmente se presentan las conclusiones.

\section{El fenómeno del dualismo en los países en desarrollo y el big push}

\subsection{La sociedad tradicional y la sociedad dual}

Las sociedades de los países con niveles de desarrollo bajo se caracterizan por la presencia de dos sectores marcadamente diferenciados: por un lado está el tradicional, que marca la etapa inicial característica de las economías preindustriales. ${ }^{3}$ En este sector las relaciones productivas se han venido perpetuando a lo largo del tiempo sin apenas variaciones y la lógica capitalista no funciona, remunerándose actividades cuya productividad marginal es nula, lo que genera un desempleo encubierto (Lewis, 1954). Este desempleo puede aparecer en cualquiera de las dos explotaciones más comunes, la familiar o la regida por un terrateniente. En la primera es consecuencia de los vínculos entre los individuos (personas con productividad marginal nula que en un mercado capitalista tradicional no percibirían salario por su trabajo, sus familiares los incorporan al mercado laboral; esta idea se vincula al concepto de capital social); ${ }^{4}$

existe una pendiente positiva y decreciente (a mayor desarrollo, más contaminación), que a partir de un determinado estadio de desarrollo adquiere pendiente negativa (a mayor desarrollo, menor contaminación).

${ }^{3}$ En la actualidad existen muy pocos ejemplos de regiones sin desarrollo industrial alguno que permanezcan en esta situación, ya que la presencia de núcleos industriales próximos o el conocimiento de éstos es fuente de migraciones y nos lleva a la siguiente etapa, el dualismo.

${ }^{4}$ Concepto derivado de la sociología, se refiere a las conexiones entre las redes sociales en las que se mueven los individuos. Este capital tendría valor económico, al igual que el capital ambiental y 
mientras que en la segunda puede aparecer por los anhelos de estabilidad social de las clases altas (se eliminaría el desempleo como posible fuente de inestabilidad y amenaza de un sistema que les favorece) o por motivos crediticios (el terrateniente proporciona al campesino un salario que posteriormente éste le devuelve con creces cuando le resulta inevitable solicitar un crédito, donde el único prestamista es el propio terrateniente) (Azqueta, 2007). Este sector es estático y depende del medio natural para su subsistencia, lo que en principio fomenta la conservación del mismo.

Frente a este sector tradicional está el sector moderno y urbano, con una dinámica optimizadora. Tal sector se caracteriza por el uso intensivo de recursos y factores, a fin de maximizar la utilidad individual. Su crecimiento dentro de la economía en las etapas iniciales de desarrollo marca el paso de una economía tradicional a una dual, ${ }^{5}$ con una industria incipiente (fase de industrialización).

El sector moderno, eficiente e intensivo en el uso del factor capital, asimila a medida que crece la mano de obra proveniente del sector rural, así se produce un traslado de factores (que genera las migraciones campociudad). Esto produce cambios en el sector tradicional, que adopta los principios de división de trabajo y especialización productiva, se empieza a valorar la tierra como un factor productivo más, y no como un garante de estabilidad. Como resultado se origina un proceso de diferenciación social y marginación, en el cual los terratenientes se transforman en empresarios que reequilibran su balanza de inputs, lo que desplaza a los campesinos y los priva en numerosas ocasiones del disfrute de recursos antaño comunes (bosques, pastos) (Azqueta, 2007). La desigualdad se acentúa también en el género: los varones jóvenes emigran y las mujeres (a menudo a cargo de sus hijos) son quienes permanecen en el medio rural. Debido a que en la ciudad los salarios son mejores, en teoría esta emigración se mantiene hasta que se genera un desempleo suficiente en las zonas urbanas como para desincentivar el desplazamiento (Harris y Todaro, 1970); no obstante, la evidencia empírica nos muestra una masificación y un elevado desempleo en los principales núcleos urbanos de los países en vías de desarrollo, que se contiene gracias al papel que desempeña el sector informal, que a la vez contiene la inmigración rural y es el sustento de los desempleados urbanos. Existe por tanto un trade-off en la transición de una economía tradicional a una economía dual, cuyas repercusiones dependen de la capacidad de la región para alcanzar un

el capital físico, al proporcionar a los integrantes de la red social un flujo de servicios de los que se obtiene una utilidad.

${ }^{5}$ La mayoría de países atrapados en equilibrios de bajo nivel están en esta situación. Un ejemplo son gran parte de los Estados subsaharianos en la actualidad. 
equilibrio superior a través de un big push ${ }^{6}$ (Rosenstein, 1943). Finalizar este proceso con éxito supone alcanzar la condición de nación desarrollada desde una perspectiva económica. ${ }^{7}$

Este proceso de transición de una economía tradicional a una economía dual tiene efectos ambientales: en el mundo rural los nuevos empresarios agrícolas adaptan técnicas más intensivas y nocivas para el medio ambiente (pesticidas y nuevas variedades genéticas que merman la biodiversidad, explotación de los acuíferos, ríos y lagos y daño a mbiental derivado de la construcción de nuevas infraestructuras); la población desplazada por el empresariado rural que permanece en este medio tiene que trabajar y vivir de las tierras marginales y los recursos comunes (Hardin, 1968), acentuando los procesos de erosión, la presión sobre los pastos y la pérdida de bosques tropicales; y quienes colonizan nuevas tierras - con frecuencia con el apoyo del gobierno, que busca mitigar así la presión migratoria y la desigualdad- contribuyen al proceso de deforestación, introducción de especies no autóctonas invasivas, sobreexplotación de ríos y lagos, etcétera.

En el sector urbano los efectos también son relevantes. La mayoría se generan por el carácter informal de los nuevos asentamientos, que al principio pueden causar la pérdida de ecosistemas valiosos, a lo que hay que añadir el agotamiento de las fuentes de agua y la contaminación hídrica, el problema de los residuos (insalubridad, infecciones, epidemias, contaminación local), la contaminación atmosférica (tanto externa como en el interior de la vivienda), el agravamiento de los desastres naturales (se reduce la permeabilidad del suelo y el proceso erosivo en laderas, lo que genera aludes de lodo), así como el incremento de la demanda y con ella la presión sobre el medio natural derivado de las mayores necesidades de consumo de la nueva clase media.

Un deterioro de estas características (acelerado y extensivo, que prácticamente llega a toda la geografía del país, y que es intensivo en las zonas urbanas) no es sustentable en el mediano y largo plazos. Una región atrapada en un equilibrio de bajo nivel como el descrito tendría elevadas tasas de descuento que favorecerían el desplazamiento del consumo al presente y un alto ritmo de deterioro de la calidad ambiental, adoptando de facto una dinámica similar a la descrita en los modelos de tipo cake eater (Hotelling, 1932; Dasgupta y Heal, 1979; Krautkraemer, 1985), en

${ }^{6}$ India o China estarían en este grupo de países.

${ }^{7}$ El término nación desarrollada es complejo y abarca gran cantidad de criterios. En función del criterio empleado, se puede considerar a un país dentro de este grupo o no. Los indicadores más frecuentes son el PIB per cápita, el grado de industrialización (aquí se considera el sector terciario y el cuarto sector, que abarca tecnologías de la información y la comunicación, o el I+D) y el índice de desarrollo humano (IDH). Al hablar de desarrollo económico dentro del big push, nos acercamos más a los dos primeros criterios que al tercero. 
los que el consumo y los recursos naturales tienden a cero en el límite por agotamiento. Esta situación se conoce como trampa de desarrollo.

Por otro lado, si la región consigue cifras de crecimiento lo suficientemente altas como para alcanzar el siguiente nivel de desarrollo, la propia dinámica de crecimiento económico alterará el peso relativo de la industria manufacturera a favor de los servicios y el cuarto sector, mejorará la infraestructura y servicios públicos y alterará la demanda ambiental de los individuos de la sociedad, facilitando una gestión sustentable del medio ambiente, lo que se conoce como curva de Kuznets (Grossman y Krueger, 1991). Por otra parte, puede que la economía no satisfaga los requisitos necesarios para alcanzar el siguiente nivel de desarrollo, o que tarde tiempo en alcanzarlo y el deterioro sobre los recursos naturales sea ya muy elevado9 (para un debate sobre la velocidad a la que se alcanza la fase descendente en la curva de Kuznets, véase Dasgupta et al., 2002), aunque esta última alternativa la rebaten la mayoría de los trabajos empíricos (López, 1994; Selden y Song, 1995; John y Pecchenino, 1994; John et al., 1995; McConnell, 1997). El resultado de una economía atrapada en un equilibrio de bajo nivel con altos grados de contaminación es un deterioro ambiental que reduce el capital natural y merma la capacidad de crecimiento del país (Gordon et al., 1987).

\section{2. $E l$ big push}

Una economía dual, con altas tasas de contaminación y deterioro ambiental, no es sostenible ambientalmente, por lo que un país debe alcan-

\footnotetext{
${ }^{8}$ Estos modelos establecen que para que se dé esta situación de agotamiento es necesaria una baja capacidad de regeneración del medio natural, falta de factores sustitutivos renovables y ausencia de progreso técnico. Hotelling (1932) demuestra que si se relajan estos supuestos, el modelo cake eater no funciona salvo en casos en los que existan trampas de pobreza con tasas de descuento muy elevadas que penalicen el consumo futuro y lo trasladen al presente. La modelización es como sigue:

$$
\begin{gathered}
\text { Max } W=\int_{0}^{\infty} U\left(C_{t}\right) e^{-\rho t} d t \\
\text { sujeto a: } C_{t}=R_{t} \\
\dot{N}_{t}=-R_{t} \\
C_{t}, R_{t} \geq 0, \forall t, N_{0} \text { dado }
\end{gathered}
$$

Donde $W$ representa el bienestar, $U(C)$ es la función de utilidad (que se obtiene mediante el consumo, $C$ ), $R$ es la cantidad de recursos consumidos y $N$ la dotación de recursos disponible.

${ }^{9}$ Existen cuatro posturas fundamentales en torno a la evolución que sigue la curva de Kuznets ambiental: la primera defiende que la curva crece hasta un determinado nivel, en el que se mantiene; la segunda, más pesimista, cree que si bien a medida que se crece se reduce la contaminación por determinados tóxicos, aparecen otros nuevos que aumentan la polución, con lo que la curva mantiene su pendiente positiva siempre (nuevos tóxicos); la tercera es la curva de Kuznets ambiental tradicional antes abordada; y la última, la más optimista, es la curva de Kuznets ambiental revisada, una crítica optimista de la curva de Kuznets ambiental convencional basada en la evidencia empírica, que compacta la $U$ invertida reduciendo el tiempo necesario para alcanzar una pendiente negativa en la curva (Dasgupta et al., 2002).
} 


\section{Figura I \\ El fenómeno del big push y la transición del subdesarrollo al desarrollo}

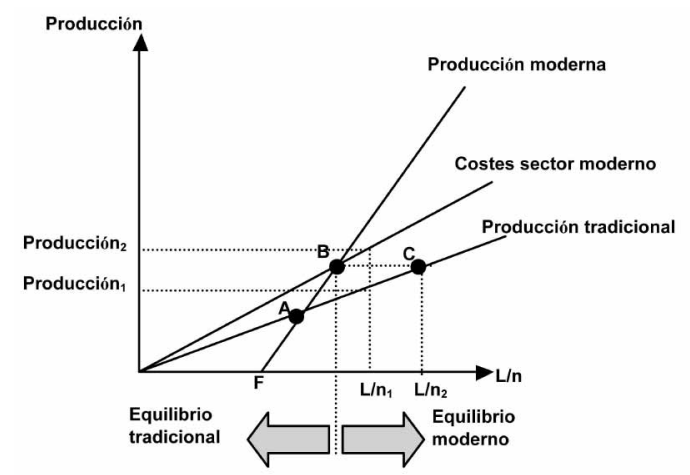

Fuente: Rosenstein (1943) y Murphy et al. (1989).

zar el siguiente estadio del desarrollo, la etapa de crecimiento sostenido que permite alcanzar un nivel de renta que garantiza la sostenibilidad ambiental. Existen diversos modelos que explican las causas de este despegue recurriendo a distintos factores desencadenantes. Nosotros nos centramos en la principal propuesta de la alta economía del desarrollo (Krugman, 1993): la teoría del big push.

Esta teoría la formuló por vez primera Rosenstein (1943). Se basaba en la fuerza de las economías externas de escala, la presencia de competencia imperfecta en la economía y en los eslabonamientos hacia delante y hacia atrás (Hirschamn, 1958); El big push establece que el desarrollo se alcanza generando las economías de escala necesarias para incrementar la capacidad productiva de sectores fuertemente interconectados con el resto de la economía para lograr un impulso colectivo. Es posible que para esto sea suficiente la iniciativa de un puñado de empresarios para iniciar la transición de la economía tradicional a la moderna; pero puede que estas iniciativas individuales dispersas no sean suficientes, y que el país necesite un verdadero despegue colectivo para entrar en la dinámica que lo conduzca al desarrollo. Es en este segundo caso en el que encontramos el problema al que se enfrentan numerosos países: es necesaria una calidad institucional suficiente para generar la base de infraestructuras, capital humano, estabilidad social, etc., necesarias para iniciar un proceso de big push. La calidad institucional y su efecto en el medio ambiente y el crecimiento lo tratamos en el apartado 2. 
La revolución emprendida por la alta economía del desarrollo tuvo críticas ${ }^{10} \mathrm{y}$ se abandonó en los años sesenta, para volver al primer plano de la economía tras el artículo de Murphy et al. (1989). Estos autores modelizaron la teoría del big push, aunque no incorporaron los mercados internacionales (figura I).

En esta figura se presenta la situación de una economía que se enfrenta a una hipotética transición hacia un equilibrio que conduce al desarrollo. Inicialmente estamos a la izquierda del punto $A$, en un equilibrio de carácter tradicional (con bajos costos y bajas productividades). Para alcanzar un equilibrio que permita la industrialización de la región se debe obtener el nivel de producción marcado por la línea de producción moderna y éste además debe ser superior a los costos. Esto implica que un esfuerzo de industrialización sólo es válido en el caso de que exista una demanda capaz de absorber la plena producción del sector y haga posible asumir los costos (los costos salariales en el sector tradicional los asumimos unitarios, mientras que en el sector moderno el salario equivale a $w>1$; hay diversas explicaciones teóricas para este salario superior, la más común es los mayores costos de la vida urbana, en términos monetarios y de desutilidad por la pérdida del capital social característico de las zonas rurales).

De esta manera, si destinamos una cantidad de factores productivos que nos sitúa a la derecha de $A$ pero a la izquierda de $B$, los costos del sector moderno superarían a los beneficios, y no sería rentable. Esto se debe a que no hemos generado economías externas de escala: la demanda sigue siendo la misma, por lo que empleando $(L / n)_{1}$ la producción seguirá siendo la que habría en el sector tradicional, producción ${ }^{\prime}$, pues no hay demanda que justifique una mayor. Por tanto, si queremos superar la barrera del big push en una economía cerrada, tendremos que emplear $(L / n)_{2}$, una cantidad mayor de trabajadores (en este caso nuestro único factor productivo), que probablemente no estén disponibles en la economía. En cambio, utilizando $(L / n)_{1}$ y a través de un esfuerzo colectivo de industrialización que permita generar economías externas de escala y crear una demanda suficiente, vemos que podríamos llegar a abastecer la cantidad producción ${ }_{2}$ sin aumentar el número de factores productivos. Por tanto, la clave está en aprovechar el multiplicador keynesiano, algo complicado si no existe un mercado desarrollado del lado de la demanda; y para generar esta demanda son necesarios salarios, lo que exige a su vez producción.

${ }^{10}$ Las críticas se referían a la falta de modelización económica y a los problemas al explicar algunos procesos de desarrollo, como el de las economías del sudeste asiático, que crecieron con base en la exportación y no en mercados internos; pero sobre todo se criticó el estancamiento de las economías en las que se pusieron en práctica políticas económicas basadas en estos principios (Krugman, 1993). 
Por tanto, el big push condiciona alcanzar una senda de crecimiento sostenido en la calidad institucional de los países en vías de desarrollo, lo que representa una barrera muy significativa para el crecimiento de estas regiones, caracterizadas en muchos casos por la inestabilidad que originan precisamente los equilibrios de bajo nivel. No obstante, si se salva esta (notable) dificultad, el big push presenta una posibilidad de alcanzar un estadio de desarrollo suficiente para garantizar la sostenibilidad ambiental y el crecimiento sostenible.

\section{3. ¿Back to the wild?}

Los problemas ambientales, sociales e institucionales inherentes al fenómeno del desarrollo y la dificultad de lograr tasas de crecimiento sostenibles en el tiempo, han motivado la aparición de corrientes contrarias a la meta del desarrollo económico per se, que defienden la necesidad de plantear una ideología del decrecimiento o acrecimiento (Latouche, 2003). Se argumenta que una vez que se descuenta el deterioro ambiental y la pérdida de capital social de las tasas de crecimiento económico, éste disminuye significativamente e incluso puede ser negativo.

Las conclusiones de los defensores del decrecimiento son fuertes (progreso técnico bajo, ausencia de factores sustitutivos renovables) y provocan que en la actualidad estas teorías no tengan mucho apoyo en las principales ramas de investigación en economía. Además, el paradigma antropocéntrico ampliado vigente, si bien internaliza aspectos más allá del interés individual del sujeto, hace inviable por ahora la posibilidad de un decrecimiento voluntario de la sociedad. Existen ejemplos de sobreexplotación y agotamiento de recursos naturales a lo largo de toda la historia, como el caso de los chinos Han ${ }^{11}$ (Landes, 1999) o el colapso de la civilización maya (Gill, 2000), por lo que la mayoría de los economistas ha optado por tratar de comprender los factores que originan estas dinámicas de sobreexplotación y actuar al respecto, antes que modificar el comportamiento de la sociedad afectada.

\footnotetext{
${ }^{11}$ Landes (1999) expone cómo los chinos Han colonizan desde los bosques limítrofes con la estepa asiática (donde quemaron los bosques y aprovecharon las tierras para la agricultura hasta su agotamiento) hasta el cauce superior del río Amarillo, donde aprenden las técnicas de la irrigación y comienzan el cultivo de arroz. Las elevadas productividades alcanzadas permiten poner en marcha un sistema capaz de sostener a comerciantes y artesanos, con base en el control del agua, en el cual se apoya en principio la autoridad imperial (talasocracia). Este ejemplo nos sirve para relativizar el mito de la sociedad tradicional respetuosa con el entorno y el medio ambiente.
} 


\section{Crecimiento y dotación de recursos naturales. El papel de las instituciones}

La mayoría de los economistas coinciden en señalar que los problemas ambientales iniciales de los países pobres (en general de carácter local o regional, como los residuos urbanos, lluvia ácida, contaminación hídrica, etc.) tienden a solucionarse una vez superados los primeros estadios de desarrollo (Grossman y Krueger, 1991; Dasgupta et al., 2002). No obstante, ya advertimos que alcanzar un nivel de desarrollo superior es un camino que no está exento de dificultades.

Muchos países con nivel bajo de desarrollo tienen elevadas dotaciones de recursos naturales, que en principio deberían constituir una ventaja comparativa frente al resto de naciones, de acuerdo con la teoría económica básica. Sachs y Warner (2001) calificaron esta aparente ventaja como la maldición de los recursos naturales, y demostraron con instrumentos econométricos que incluso controlando las variaciones en los precios de los recursos (los economistas de la Escuela de la CEPAL argumentaban que el deterioro de los términos de intercambio era el problema fundamental a combatir), la evolución del PIB en los distintos países considerados para la muestra en la década anterior, los factores geográficos, así como la abundancia de recursos naturales siguen siendo una variable significativa a la hora de explicar el PIB con un coeficiente negativo. Este argumento chocaba con la creencia general de que una abundancia relativa de recursos naturales era un determinante positivo para el crecimiento, vigente desde el trabajo de Habakkuk (1962), ${ }^{12}$ cuyas conclusiones se habían empleado como fundamento para la ejecución de políticas de desarrollo basadas en el potencial de determinadas zonas en recursos naturales.

Sachs y Warner fundamentaban este argumento en el hecho de que la abundancia de recursos naturales introduce una serie de incentivos que perjudican al sector exportador manufacturero, motor del proceso de industrialización: la relativa abundancia de recursos naturales en la economía fomenta la utilización de factores productivos en este sector, pero los encarece y merma la competitividad de las manufacturas nacionales, y con ello disminuye las exportaciones. ${ }^{13}$ El efecto es similar al de la enfermedad holandesa, con la excepción de que la maldición de los recursos naturales únicamente afecta a países con niveles bajos de desarrollo.

${ }^{12}$ De hecho Habakkuk tenía razón en creer que la abundancia de recursos naturales facilitaba el desarrollo bajo ciertos supuestos, como veremos más adelante. Además, a finales del siglo xix y principios del xx la movilidad de factores era mucho menor de lo que es hoy en día, y la presencia de recursos naturales en territorio nacional podía ser un imperativo para el desarrollo.

${ }^{13}$ Otras explicaciones se centran en variables de carácter educativo y en la falta de iniciativas empresariales como factores explicativos, pero nosotros nos remitimos a la hipótesis original de los autores. 
Ning y Field (2005) ahondan sobre el trabajo anterior y prueban que la causa del subdesarrollo en estos países no es la presencia de recursos naturales, sino la dependencia de los mismos. Demuestran que a partir de una serie de supuestos una dotación de recursos naturales comparativamente relevante afecta de forma positiva a la economía; pero si ésta es incapaz de desarrollar actividades productivas y se limita a explotar esta dotación inicial no se fomentará la exportación, innovación y mejoras productivas que conduzcan a un nivel de desarrollo alto. Los autores llegan a esta conclusión incorporando a la regresión econométrica original un indicador de dotación de recursos naturales, además del porcentaje de exportaciones de recursos naturales sobre el pIB empleado por Sachs y Warner. El resultado es que la dotación de dichos recursos tiene signo positivo, mientras que la dependencia lo tiene negativo, siendo ambos coeficientes significativos. Posteriormente generan un sistema de ecuaciones simultáneas y encuentran que ni la dotación ni la dependencia sobre los recursos naturales son significativas en el crecimiento del PIв. No obstante, Sala-i-Martin y Subramanian (2003) corrigen este argumento al demostrar que el efecto de la dotación de recursos naturales en el crecimiento del PIB es indirecto a través de la calidad institucional, que sí es una variable explicativa del crecimiento del PIB.

Por tanto, el efecto de la dotación de recursos sobre el crecimiento del PIв no es directo, sino que se produce vía calidad institucional. Mehlum et al. (2006) desarrollaron un trabajo empírico-teórico que arroja luces en esta relación: ambas variables actúan conjuntamente, por lo que el resultado de la abundancia de recursos naturales en una economía sobre el рIв dependerá en última instancia de la calidad institucional de la misma. Si el sector público promueve actividades productivas, los sectores vinculados a la explotación de los recursos naturales y los manufactureros serán complementarios, con lo que se benefician mutuamente (producer friendly); si por el contrario el sector público es ineficiente y favorece las actividades predatorias, se generará una relación de carácter sustitutivo entre ambos sectores y habrá un efecto nocivo en el PIB (grabber friendly) (figura II).

Con las instituciones idóneas, el descubrimiento de nuevos recursos naturales conducirá a un aumento de los beneficios y por ende del PIB (punto $B$, figura $\mathrm{I}$ ), gracias al desplazamiento de la curva de beneficios del productor hacia arriba. Si por el contrario el aparato estatal es ineficiente y favorece las actividades de búsqueda de rentas, el descubrimiento empeora la situación de los productores, de acuerdo con el argumento original de Sachs y Warner. La economía vería disminuir su producción a costa del enriquecimiento de los depredadores, y se trasladaría al punto C. Por tanto, la riqueza natural puede conducir a situaciones tan diversas 


\section{Figura II}

\section{Efectos del descubrimiento de nuevos recursos naturales con instituciones producer friendly y grabber friendly}

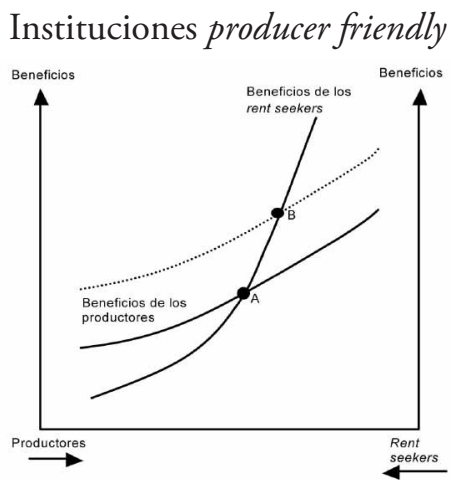

Instituciones grabber friendly

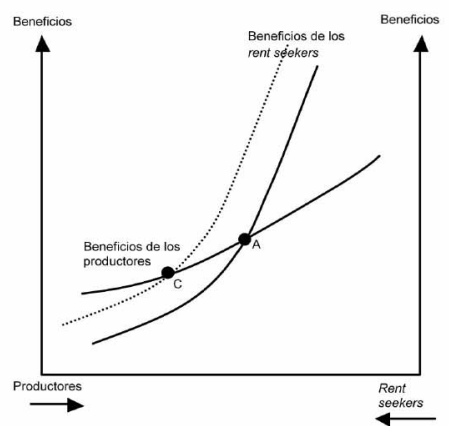

Fuente: Mehlum et al. (2006).

como las que observamos en el mundo real, con casos como los de Estados Unidos y Noruega por un lado, y los de Nigeria, Sierra Leona o el Congo en el extremo opuesto. Todo dependerá del desarrollo institucional de cada país.

Esta conclusión enlaza directamente con la teoría institucional, en la que destacan las aportaciones de autores como Daron Acemoglu, Douglas North o Kenneth Galbraith. Acemoglu et al. (2004) exponen los efectos que tiene sobre el crecimiento económico la calidad institucional de cada país, ${ }^{14}$ estableciendo la dotación de recursos naturales y la clase de recursos naturales disponibles en el país como un factor explicativo de la

${ }^{14} \mathrm{El}$ artículo analiza dos experimentos históricos (Corea del Norte frente a Corea del Sur y América del Norte frente a América del Sur y Central) y determina que el resultado final dependerá de las fuerzas iniciales y la evolución de los poderes de iure (legal, el Estado) y de facto (agentes económicos), cuya dinámica estará condicionada por la regulación estatal y las presiones ejercidas por los grupos con intereses económicos. Cuando las instituciones definan y fortalezcan los derechos 
misma. En concreto analiza un experimento natural: compara la evolución de América del Norte y América del Sur y Central. Los autores establecen como condición crucial para el correcto desarrollo institucional la ausencia de rentas de las cuales apropiarse. Se da como ejemplo la conquista de la relativamente desarrollada sociedad azteca frente a la de las tribus norteamericanas; mientras en México el oro y la minería se convirten en la actividad principal, en Norteamérica los vastos territorios vírgenes no ofrecen estas posibilidades, lo que reduce las posibilidades de obtener beneficios apropiándose de las rentas y potencia una protoindustrialización como la que se llevó a cabo.

Los países atrapados en trampas de pobreza con elevadas dotaciones de recursos naturales dependen en gran medida de éstos para obtener rentas, lo que acentúa la presión sobre el medio ambiente y limita las posibilidades de desarrollo futuro. Hemos tratado el papel de las instituciones para encauzar el crecimiento que permita un desarrollo sostenible, que la bibliografía coincide en resaltar. Sin embargo, existen otros factores que afectan al crecimiento y la calidad ambiental, temas que tratamos en el siguiente apartado.

\section{Crecimiento y calidad ambiental: otros factores explicativos}

\subsection{La geografía}

La geografía es un factor a tener en cuenta en la transición a los estadios de desarrollo económico. Se ha tendido a subrayar las ventajas de Europa en cuanto a temperatura (inviernos lo bastante fríos como para acabar con los patógenos, pero no lo suficientemente fríos como para acabar con la agricultura durante estos meses) o régimen de lluvias (muy regular, con escasos episodios de sequía extrema o inundaciones; esto además evitó que aparecieran regímenes autocráticos de poder basados en el control de este recurso, como sucedió en China). También existen desventajas: Europa tiene un desarrollo tardío, explicado en parte por la dificultad de colonizar zonas boscosas sin herramientas de hierro; además, existía menor productividad por unidad de tierra que en otras zonas más fértiles, donde al principio la agricultura se desarrolló mejor (Nilo, Éufrates, Indo). Pero las ventajas, una vez superada la etapa inicial de subdesarrollo, favorecieron un crecimiento rápido y sostenido a lo largo del tiempo (Landes, 1999).

de propiedad, establezcan límites eficientes a los entes políticos reguladores y cuando haya relativamente pocas rentas de las que apropiarse se podrá encauzar un crecimiento adecuado y sostenible. 


\subsection{Información imperfecta y estratificación de la cadena productiva}

Existen asimetrías en la información que perjudican a los estratos más vulnerables de la sociedad en los países en desarrollo, especialmente en el medio rural. El desconocimiento de los mercados y la falta de organización provocan que los intermediarios se apropien de parte de la renta por prestar servicios que podría desarrollar la comunidad. En ocasiones esta pérdida se compensa a través de una mayor presión sobre el medio natural.

\subsection{Deterioro de los términos de intercambio}

La Escuela de la CEPAL señaló éste como el principal problema que deben afrontar los países en desarrollo para lograr el crecimiento, aunque ya vimos en el apartado anterior que esta importancia ha disminuido. No obstante, sí es destacable el efecto que la pérdida relativa de valor de las exportaciones de estos países (en gran medida productos primarios y recursos naturales) en relación con las importaciones (terms of trade o términos de intercambio) tiene sobre el medio ambiente, al incrementar la presión sobre los activos ambientales exportados para mantener una balanza comercial equilibrada.

\subsection{Dumping ambiental}

El modelo del big push parte de una economía cerrada. Pero el comercio internacional es una variable clave para explicar el desarrollo vía exportaciones, así como un causante del deterioro ambiental (Azqueta et al., 2006).

Las distintas regulaciones generan ventajas comparativas que pueden derivar en una explotación extensiva de los recursos naturales (Azqueta y Sotelsek, 1999). El dumping ambiental en especial se concentra en las fases iniciales del desarrollo, aunque después remite (curva de Kuznets). Este fenómeno aparece cuando empresas internacionales se instalan en países en desarrollo para beneficiarse de una normativa ambiental menos exigente (Rauscher, 1994). El dumping es un fallo de mercado en el que la sociedad receptora alcanza un óptimo privado, alejándose del óptimo social (daño ambiental, enfermedades, insalubridad y suciedad son algunas consecuencias) (Gradus y Smulders, 1993).

También se ha defendido que el dumping, a través del posicionamiento estratégico de empresas extranjeras, permite lograr el impulso que 


\section{Figura III}

\section{La teoría pura del comercio internacional}

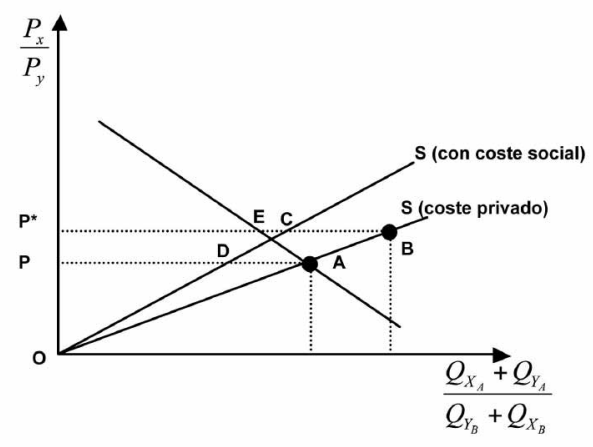

Fuente: Anderson y Blackhurst (1992).

permite al país alcanzar el desarrollo sostenible ${ }^{15}$ (Copeland y Taylor, 1994). En este caso, se sacrifica la generación actual a cambio de un mayor bienestar futuro. La teoría pura del comercio internacional establece que una vez que se aprovecha esta ventaja comparativa el daño ambiental es irreversible (cake eater), mientras que la nueva teoría del comercio internacional asume preferencias dinámicas cambiantes que favorecen crecimiento y sostenibilidad (curva de Kuznets).

La teoría pura del comercio internacional, basada en la ventaja comparativa ricardiana, predice el dumping ambiental. Los países en desarrollo emplean el medio ambiente como ventaja comparativa. El problema de la teoría ricardiana es que las preferencias son estáticas, por lo que el deterioro ambiental es continuo (Anderson y Blackhurst, et al., 1992) (figura III).

Cantidades y precios aparecen en términos relativos (bienes de un país frente a bienes del resto del mundo y precios de un bien frente a precios del otro). $A$ es un país en desarrollo, y $B$ es el resto del mundo. Hay dos bienes, $x$ e $y$. Si la relación internacional de precios es $P^{*}$, mientras que el cociente nacional es $P$ (el bien $x$ es relativamente más barato), el país $A$ tendrá la oportunidad de ofrecerlo en el mercado internacional, incrementando su producción hasta el punto $B$. Con una normativa ambiental laxa en la que no internalicemos costos externos al mercado, el impacto ambiental que habremos de afrontar aumentará del área formada por el triángulo $O D A$ a aquélla formada por $O B C$. Por tanto,

${ }^{15}$ Los efectos del dumping ambiental sobre las tasas de crecimiento de la economía dependen de numerosos factores. Como veremos en el penúltimo bloque, hay países como China e India que han compatibilizado regulaciones ambientales laxas con un mayor PIB y existencias de capital (incluidos aquí capital humano, natural y físico conjuntamente), mientras que en otros casos el resultado ha sido el inverso (Arrow et al., 2004). 
el deterioro ambiental será mayor del que existiría en una economía autárquica, a cambio de un mayor bienestar (área $E B A$ ).

La asunción de preferencias estáticas es poco razonable, por lo que se desarrolla la nueva teoría del comercio internacional (Krugman, 2006), que parte de modelos como el big push. Esta teoría tiene raíces empíricas en el incremento de los intercambios intraindustriales (y no interindustriales) que se dieron con la creación de la UE, algo que no predecían HeckscherOhlin-Samuelson. El modelo incorpora la competencia monopolística, las externalidades y relaja la asunción de rendimientos constantes a escala (aparecen las economías de escala propias del big push). Bajo estos supuestos, se consideran preferencias dinámicas que permiten un cambio en la ventaja comparativa a lo largo del tiempo y en las distintas etapas del desarrollo, de manera que el dumping ambiental deja de ser irreversible.

Las dos teorías son compatibles en un esquema de desarrollo dinámico: la teoría pura del comercio internacional se adapta a las fases iniciales, mientras que la nueva teoría del comercio internacional ofrece una buena modelización de cómo se produce el estadio de despegue y consolidación.

\section{Medidas paliativas frente al deterioro ambiental en ausencia de crecimiento}

Existen numerosos países en trampas de pobreza. Además, algunos de los que crecen lo hacen a costa de un fuerte deterioro ambiental en etapas de desarrollo intermedio que pueden durar décadas. Por ello se han propuesto medidas paliativas ${ }^{16}$ que buscan limitar los efectos en el medio ambiente aun en ausencia de desarrollo económico:

1. Certificación ambiental y comercio justo: el comercio justo es una alternativa al comercio convencional. Se fija un precio que no refleja únicamente el valor del producto en cuestión, sino que actúa como garantía para el productor, cumpliendo la función de aseguradora (así se ofrece un servicio que no está disponible en los países productores debido al escaso desarrollo del sector crediticio). La certificación ambiental es una señal de que el proceso productivo cumple con determinados estándares previamente establecidos; el problema de este sistema es que corre a cuenta del productor, lo que hace que este procedimiento se siga poco en los países de renta baja.

\footnotetext{
${ }^{16}$ Estas medidas, si bien pueden ser útiles, no son una solución al problema ambiental, que únicamente se puede salvar llegando a las etapas más avanzadas del desarrollo.
} 
2. Reducción del proteccionismo del sector agrario: el objetivo es que los productores del Tercer Mundo puedan beneficiarse de un mayor mercado y más excedente. Si bien esta propuesta tiene consistencia teórica y ofrece beneficios concretos (entrada de divisas, lo que limita la presión sobre los recursos naturales), éstos pueden tener una contraprestación importante en forma de deforestación. Además, la actual coyuntura internacional en los mercados de alimentos cuestiona la fiabilidad de estas economías como proveedores estables.

3. Pago de servicios ambientales (PSA): los beneficiarios de la calidad ambiental se identifican en los países en desarrollo y se les pide pagar parte de los costos o el costo de oportunidad en los que están incurriendo estos países subdesarrollados para ofrecerlas. Cuando los costos no se corresponden con los beneficios (en general estos últimos siempre se declararán menores, independientemente de si lo son o no), entra en marcha la Global Environment Facility (GEF, 2009) que trata de compensar inyectando la diferencia monetaria.

4. Deuda ecológica: se defiende que el actual intercambio ambiental entre países desarrollados y en desarrollo no es justo (los primeros están pagando un precio inferior al ecológicamente correcto, dumping, explotación de los recursos naturales para extraer divisas, etc.) (Rees, 2000). No existe un mecanismo de compensación claro, debido a la variedad de criterios. Si seguimos el principio de quien contamina paga, los candidatos a recibir las transacciones serían países como Indonesia, Tanzania, Brasil o Argentina, pero en la columna del debe encontraríamos a países como China o India, y es poco justificable desde el punto de vista de la equidad solicitar pagos a estas naciones para satisfacer el superávit ecológico de Finlandia o Canadá.

Estos son algunos de los métodos que se presentan para cubrir el deterioro ambiental a través de distintos mecanismos. Su viabilidad y eficacia han sido discutidas, pero son una alternativa a la explotación sin límites del medio ambiente.

\section{5. ¿Qué nos depara el futuro?}

El desarrollo es un fenómeno muy complejo, con frecuencia medido a través de un indicador de la renta como el PIB per cápita. Se ha criticado éste y otros indicadores de flujo por su incapacidad para actuar como variables proxy del bienestar, que es el indicador con verdadera relevancia para la economía. Autores como Dasgupta (2009) y Arrow et al. (2004) 
proponen emplear indicadores de riqueza de la economía para medir este bienestar. La riqueza genuina es un indicador que incorpora todos los activos de capital de la economía en el momento $t$, lo que incluye capital natural, físico y humano. Una economía es sostenible en $t$ si la riqueza genuina no disminuye, es decir, si la inversión genuina es positiva. ${ }^{17}$

Arrow et al. (2004) analizan la riqueza genuina de diversas regiones con datos del Banco Mundial para el ańo 2003. Se encuentra que la inversión genuina es negativa en regiones en desarrollo como el África subsahariana, Oriente Medio y el Magreb. Tomando como referencia la riqueza genuina per cápita (una vez ajustada al crecimiento poblacional), se encuentra que el aumento de la riqueza genuina en estas regiones es negativo y se sitúa muy por debajo de la tasa que refleja el рів. En estas regiones la destrucción de capital afecta las posibilidades de crecimiento negativamente, y con ello el valor presente del bienestar social.

Estos datos nos llevan de nuevo al argumento inicial: antes de debatir sobre el derecho a contaminar de determinadas regiones, es preciso evaluar el efecto que esta contaminación tiene en la tasa de crecimiento de los países. Hemos visto que a partir de distintas coyunturas el deterioro ambiental puede venir acompańado de crecimiento o es posible suponer que empeore en relación con la situación inicial. Es necesario analizar la dinámica de los factores que intervienen en el desarrollo para anticipar los efectos que el uso intensivo de los recursos naturales tiene en distintas circunstancias.

\section{Conclusión}

En este trabajo se defiende la hipótesis de que el desarrollo es necesario para garantizar la sostenibilidad ambiental en los países de renta baja. Las condiciones para que un país abandone un equilibrio de bajo nivel son, sin embargo, altamente exigentes, y requieren un esfuerzo colectivo muy difícil de coordinar. Repasamos la literatura de la maldición de los recursos naturales para resaltar el papel que éstos pueden jugar en la consolidación de un nivel de bienestar alto una vez que se ha superado la fase de transición, y vinculamos los problemas que la abundancia puede generar en las etapas iniciales de desarrollo cuando las instituciones no están lo suficientemente desarrolladas. Hemos tratado de profundizar en uno de los aspectos que

${ }^{17}$ La variación de la utilidad social intertemporal ( $V$, o valor presente del flujo de utilidades futuras) es positiva si la inversión lo es (Arrow et al., 2004):

$$
\frac{\partial V}{\partial t}=\sum_{i}\left(\partial V / \partial K_{i t}\right)\left(\partial K_{i t} / \partial t\right)=\sum_{i} p_{i t} I_{i t}
$$

Donde $V$ es la utilidad social intertemporal, $t$ denota el tiempo, $K$ la dotación de capital, $p$ el nivel de precios e $I$ la inversión. 


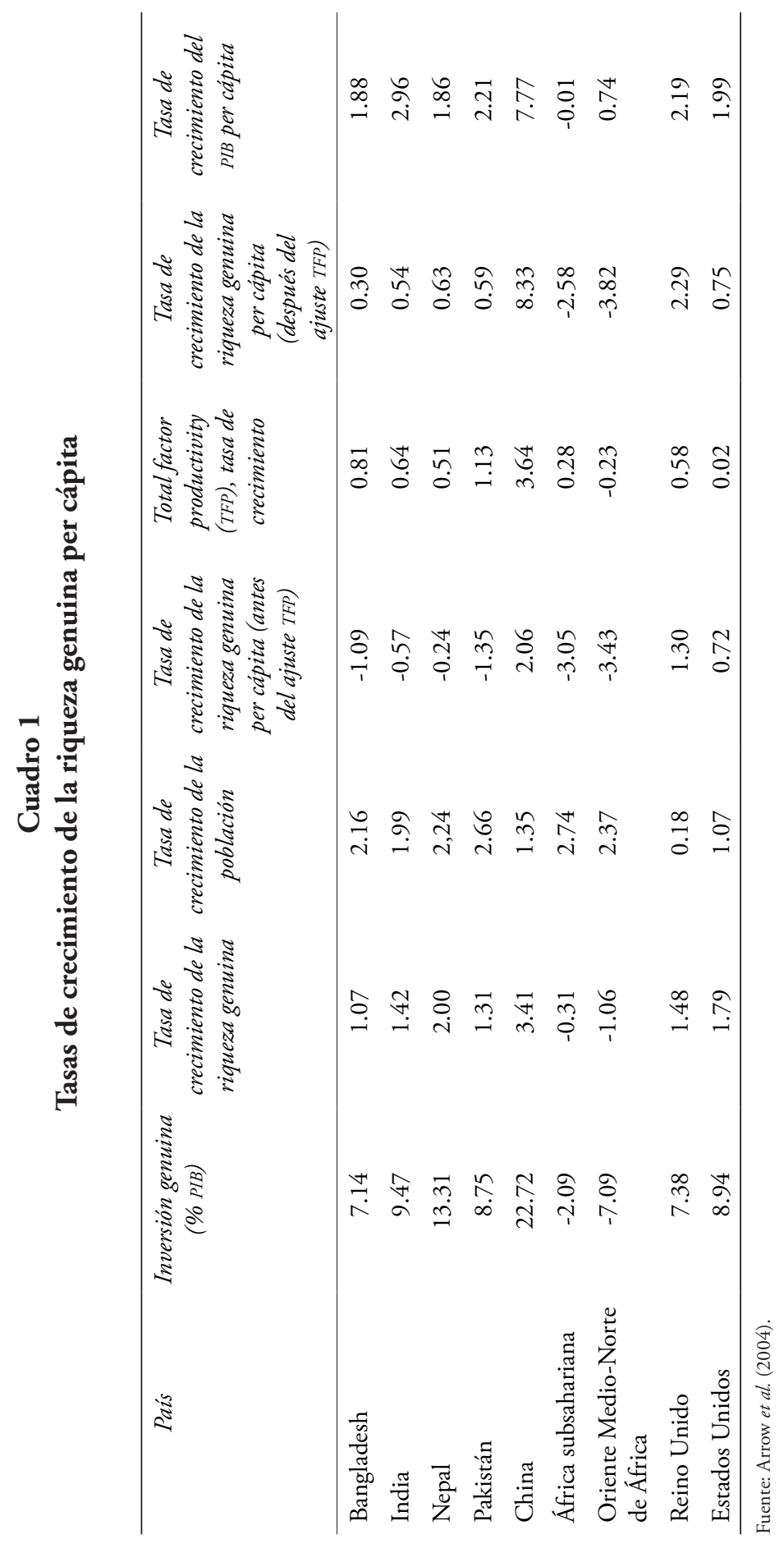


más inquietan a los economistas dedicados al estudio de la problemática ambiental, el del comercio y el aprovechamiento de ventajas comparativas de tipo ambiental. De nuevo en este apartado hemos tratado de demostrar que estas ventajas comparativas indeseables deben eliminarse saltando a una nueva trayectoria que nos conduzca a un equilibrio de alto nivel, en el que existan las condiciones necesarias para generar nuevas ventajas comparativas a través de economías de escala y eslabonamientos. Para explicar la dinámica a seguir en este proceso, utilizamos como base teórica determinadas ideas del big push que recientemente han vuelto al primer plano del pensamiento económico de la mano de Paul Krugman, quien acuñó el término alta teoría del desarrollo con el que nos hemos referido a este cúmulo de planteamientos antańo inconexos e imposibles de modelizar, pero que hoy en día se están incorporando a diversas teorías vigentes para suavizar determinadas asunciones y dar paso a nuevas ideas e incluso nuevas teorías (como hemos comprobado).

Finalmente, hemos insistido en la importancia de generar desarrollo para frenar el deterioro notable y sostenido en que están incurriendo determinadas regiones subdesarrolladas y examinamos las alternativas energéticas que se están barajando para conseguirlo, insistiendo en la polémica del biodiésel y abriendo una ventana a la esperanza que siempre ofrece la innovación tecnológica.

\section{Bibliografía}

Acemoglu, Daron, Simon Johnson y James Robinson (2004), "Institutions as the Fundamental Cause of Long-Run Growth”, NBER Working Paper, W10481, National Bureau of Economic Research, Cambridge.

Anderson, Kym y Richard Blackhurst (1992), The Greening World Trade Issues, Harvester Wheatsheaf, Londres.

Arrow, Kenneth, Partha Dasgupta, Lawrence Goulder, Gretchen Daily, Paul Ehrlich, Geoffrey Heal, Simon Levin, Karl-Goran Maler, Stephen Schneider, David Starrett y Brian Walker (2004), "Are we consuming too much?”, Journal of Economic Perspectives, 18 (3), American Economic Association, Nashville, pp. 147-172.

Azqueta-Oyarzún, Diego (2007), Introducción a la economía ambiental, McGraw-Hill, Madrid. 
Azqueta-Oyarzún, Diego, Gonzalo Delacámara y Daniel Sotelsek (2006), "Degradación ambiental, endeudamiento externo y comercio internacional”, Cuadernos Económicos del ICE, 71, Ministerio de Industria, Comercio y Turismo de España, Madrid, pp. 115-132.

Azqueta-Oyarzún, Diego y Daniel Sotelsek (1999), "Ventajas comparativas y explotación de los recursos ambientales", Revista de la CEPAL, 68, Comisión Económica para América Latina y el Caribe, Santiago de Chile, pp. 115-134.

Copeland, Brian y Scott Taylor (1994), "North-South Trade and the Environment", Quarterly Journal of Economics, 109 (3), Massachusetts Institute of Technology, Cambridge, pp. 755-787.

Dasgupta, Partha y Geoffrey Heal (1979), Economic Theory and Exhaustible Resources, Cambridge University Press, Cambridge.

Dasgupta, Partha (2009), "The Welfare Economic Theory of Green National Accounts", Environmental and Resource Economics, 42 (1), Cambridge University Press, Cambridge, pp. 3-38.

Dasgupta, Susmita, Benôite Laplante, Hua Wang y David Wheeler (2002), "Confronting the Environmental Kuznets Curve", The Journal of Economic Perspectives, 16 (1), American Economic Association, Nashville, pp. 147-168.

GEF (Global Environment Facility) (2009), Annual Performance Report, Global Environment Facility, Washington.

Gill, Richardson (2000), The Great Maya Droughts, University of New Mexico Press, Albuquerque.

Gómez-Gómez, Carlos Mario (2007), "The Modere Model and The Economic Analysis of Farmer's Decisions", borrador.

Gordon, Robert, Tjalling Koopmans, William Nordhaus y Brian Skinner (1987), Toward a New Iron Age? Quantitative Modelling of Resource Exhaustion, Harvard University Press, Harvard.

Gradus, Raymond y Sjak Smulders (1993), “The Trade-off between Environmental Care and Long-term Growth-Pollution in Three 
Prototype Models", Journal of Economics, 58 (1), Springer, Berlín, pp. 25-51.

Grossman, Gene y Alan Krueger (1991), "Environmental Impacts of a North American Free Trade Agreement", CEPR Working Paper, 644, Centre for Economic Policy Research, Londres.

Habakkuk, John (1962), American and British Technology in the Nineteenth Century, Cambridge University Press, Cambridge.

Harbaugh, William, Arik Levinson y David Wilson (2002), "Reexamining the Empirical Evidence for an Environmental Kuznets Curve", Review of Economics and Statistics, 84 (3), Massachusetts Institute of Technology, Cambridge, pp. 14-28.

Hardin, Garrett (1968), "The tragedy of the commons", Science, 162, American Association for the Advancement of Science, Washington, pp. 1243-1248.

Harris, John y Michael Todaro (1970), "Migration, Unemployment \& Development: A Two-Sector Analysis", American Economic Review, 60 (1), American Economic Association, Nashville, pp. 126-142.

Hirschman, Albert O. (1958), The Strategy of Economic Development, Yale University Press, New Haven.

Hotelling, Harold (1932), "Edgeworth's taxation paradox and the nature of demand and supply functions", Journal of Political Economy, 40, University of Chicago, Chicago, pp. 577-616.

John, Andrew y Rowena Pecchenino (1994), "An Overlapping Generations Model of Growth and the Environment", Economic Journal, 104 (427), Wiley Blackwell, New Jersey, pp. 1393-1410.

John, Andrew, Rowena Pecchenino, David Schimmelpfennig y Stacey Schreft (1995), "Short-Lived Agents and the Long Lived Environment”, Journal of Public Economics, 58 (1), Elsevier, Cambridge, pp. 127-141. 
Krautkraemer, Jeffrey (1985), "Nonrenewable resource scarcity”, Journal of Economic Literature, 36 (4), American Economic Association, Nashville, pp. 2065-2107.

Krugman, Paul (1993), “Towards a Counter-Counterrevolution in Development Economics", en Lawrence Summers y Shekhar Shah (eds.), Preceedings of the World Bank Annual Conference on Development Economics 1992, Banco Mundial, Washington.

Krugman, Paul y Maurice Obstfeld (2006), Economía internacional. Teoría y politica, Pearson, Madrid.

Landes, David (1999), The Wealth and Poverty of Nations: Why Are Some So Rich and Others So Poor?, W.W. Norton, Nueva York.

Latouche, Serge (2003), Decrecimiento y posdesarrollo, El Viejo Topo, Madrid.

Lewis, Arthur (1954), "Economic Development with Unlimited Supplies of Labour", Manchester School of Economic and Social Studies, 22 (2), Blackwell Publishing, New Jersey, pp. 139-191.

López, Ramón (1994), "The Environment as a Factor of Production. The Effects of Economic Growth and Trade Liberalization", Journal of Environmental Economics and Management, 27 (2), Elsevier, Cambridge, pp. 163-184.

McConnel, Kenneth (1997), "Income and the Demand for Environmental Quality”, Environmental and Development Economics, 2 (4), Cambridge University Press, Cambridge, pp. 383-399.

Mehlum, Halvor, Karl Moene y Ragnar Torvik (2006), "Cursed by Resources or Institutions?”, World Economy, 29 (8), Blackwell Publishing, New Jersey, pp. 1117-1131.

Murphy, Kevin, Andrei Shleifer y Robert Vishny (1989), "Industrialization and the Big Push", Journal of Political Economy, 97 (5), University of Chicago, Chicago, pp. 1003-1026.

Ning, Ding y Barry Field (2005), "Natural Resource Abundance and Economic Growth", Land Economics, 81 (4), University of Wisconsin Press, Madison, pp. 496-502. 
ONU (Organización de las Naciones Unidas) (2005), Millennium Ecosystem Assessment: Ecosystems and Human Wellbeing: Synthesis, Island Press, Nueva York.

ONU (Organización de las Naciones Unidas) (2008), Composition of macro geographical (continental) regions, geographical sub-regions, and selected economic and other groupings, United Nations Statistics Division, Nueva York.

Rauscher, Michael (1994), "On Ecological Dumping”, Oxford Economic Papers, 46 (0), Oxford University Press, Oxford, pp. 822-840.

Rees, William (2000), "Ecological footprint: merits and brickbats", Ecological Economics, 32 (3), Elsevier, Cambridge, pp. 371-374.

Rosenstein-Rodan, Paul (1943), "Problems of Industrialization of Eastern and South-Eastern Europe”, Economic Journal, 53 (210-211), Wiley Blackwell, New Jersey, pp. 202-211.

Sachs, Jeffrey y Andrew Warner (2001), "The curse of natural resources", European Economic Review, 45 (4-6), Elsevier, Berlín, pp. 827838.

Sala-i-Martin, Xavier y Arvind Subramanian (2003), "Addressing the Natural Resource Curse: An Illustration from Nigeria”, NBER Working Paper, 9804, National Bureau of Economic Research, Cambridge.

Selden, Thomas y Daqing Song (1995), "Neoclassical Growth, the JCurve for Abatement, and the Inverted U Curve for Pollution", Journal of Environmental Economics and Management, 29 (2), Elsevier, Cambridge, pp. 162-168.

Syed, Munir Khasru y Muaz Jalil Mohammad (2004), "Revisiting Kuznets Hypothesis: An Analysis with Time Series and Panel Data", Bangladesh Development Studies, 30 (3-4), Bangladesh Institute of Development Studies, Dhakar, pp. 89-112.

Recibido: 20 de noviembre de 2009.

Reenviado: 22 de mayo de 2010. Aceptado: 18 de enero de 2011. 
Carlos Dionisio Pérez-Blanco. Es licenciado en economía por la Universidad de Salamanca; es máster en economía del desarrollo (Università di Roma, Tor Vergata) y en análisis económico aplicado (Universidad de Alcalá). Actualmente es investigador contratado en el Estudio Madrileńo de Estudios Avanzados para el Agua (IMDEA-Agua) y profesor asistente en la Universidad de Alcalá, donde cursa sus estudios de doctorado. Sus líneas de investigación son economía del agua, economía ambiental y economía del desarrollo. Entre sus últimas publicaciones destacan: en coautoría, "El riesgo de disponibilidad de agua en la agricultura: una aplicación a las cuencas del Guadalquivir y del Segura", Estudios de Economía Aplicada, 1 (29), Universidad de Valladolid, Valladolid, pp. 1-26 (2011); en coautoría, "Cambio estructural regional y agua: escasez, dependencia e impactos sobre el tejido económico. El caso de Andalucía”, Estudios de Economía Aplicada, 2 (28), Universidad de Valladolid, Valladolid, pp. 423-446 (2010); en coautoría, Informe sobre la Cooperación Internacional al Desarrollo en Castilla y León, 2005-2007. Un proceso en crecimiento, Fundación General de la Universidad de Valladolid, Valladolid, 353 pp. (2010). 